\title{
Vision in chicks with distorted visual fields
}

NEVILLE MORAY AND ANNE JORDAN

DEPARTMENT OF PSYCHOLOGY, UNIVERSITY OF SHEFFIELD, ENGLAND

In a repeat of the experiment by Hess in which chicks were reared wearing hoods which bore prisms to distort the visual field by imposing a right deviation to it, we have found such animals do not show a systematic displacement of their pecks. On the other hand, they become progressively less accurate in their pecking.

Hess (1956) placed hoods carrying prisms on newly hatched chicks, causing a displacement of $7^{\circ}$ in the apparent position of objects in the visual fleld. The animals pecked at grains of corn and at bright nail heads embedded in modelling clay. By observing the beak marks in the clay Hess concluded that while the scatter of pecks decreased over the several days of the experiment, the birds did not learn to offset their pecks to compensate for the displacement of the visual field, and that therefore the space perception of the chick was innate.

One possible explanation for the failure of Hess birds to compensate is that with $7^{\circ}$ displacement so few hits are made on the grains of corn that the birds are not reinforced often enough for making the correct response, and therefore no learning occurs. To investigate this we have repeated the experiment using a series of prisms, and otherwise matching the experiment as closely as possible, with the exception that the animals used were Sussex Rhodes, and not Leghorns as used by Hess (1956).

The chicks were hatched in an incubator and at once placed in a dimly illuminated environment. As soon as they were dry and standing they were placed in the hoods. The hoods were made of muslin and rubber solution and were much lighter than pure rubber ones. The prisms were cut to our specification by the optical instrument makers Bausch and Lomb. The deviations produced by the prisms were $0^{\circ}, 2^{\circ}, 4^{\circ}, 6^{\circ}$, and $8^{\circ}$. Each deviation was used on three birds. Both prisms were mounted with their base to the left.

The number of animals is very small because the above experiment was intended as a pilot experiment for the main investigation, in which birds would be moved progressivelv from $0^{\circ}$ through the series to the $8^{\circ}$ displacement, thus allowing learning to proceed progressivly. The trials consisted of $10-\mathrm{min}$ periods in an arena whose floor was covered in modelling clay in which bright nail heads were embedded. We included controls for hunger, development of peck- ing response, etc., to parallel those mentioned by Hess. Percentage of hits and misses are shown in Table 1. The group with no displacement was considerably more accurate (63\% hits) than the groups with displacement ( $24 \%$ for $2^{\circ}$ and $21 \%$ for $8^{\circ}$ ), as might be expected. However, although all groups tended to peck short, the displacement groups did not, as in Hess's experiment, peck predominantly to one side at a distance corresponding to the displacement of the prisms. This, on the other hand, did not constitute learning to compensate for the displacement, for they became less accurate as the trials proceeded. They also pecked more.

The interpretation of these results is not clear. They do not confirm Hess's finding of a constant error corresponding to the displacement of the prisms. On the other hand, they do not indicate any successful compensation for displacement.

Table 1 Hits and misses to left and right of target at the least and greatest displacements

\begin{tabular}{|c|c|c|c|c|c|c|c|c|c|}
\hline \multicolumn{2}{|c|}{ Displacement } & \multicolumn{2}{|c|}{$0^{\circ}$} & \multicolumn{3}{|c|}{$2^{\circ}$} & \multicolumn{3}{|c|}{$8^{\circ}$} \\
\hline \multirow{3}{*}{$\frac{\text { Trials }}{1}$} & \multirow{3}{*}{$\frac{\text { Hits }}{7}$} & L. & R. & \multirow{3}{*}{$\frac{\text { Hits }}{1}$} & \multirow{2}{*}{\multicolumn{2}{|c|}{$\begin{array}{c}\text { L. R. } \\
\text { Misses }\end{array}$}} & \multirow{3}{*}{$\frac{\text { Hits }}{0}$} & \multirow{2}{*}{\multicolumn{2}{|c|}{$\begin{array}{l}\text { L. } \quad \text { R. } \\
\text { Misses }\end{array}$}} \\
\hline & & \multicolumn{2}{|c|}{ Misses } & & & & & & \\
\hline & & 0 & 0 & & 0 & 0 & & 0 & 0 \\
\hline 2 & 23 & 0 & 2 & 1 & 0 & 0 & 12 & 5 & 7 \\
\hline 3 & 3 & 1 & 3 & 3 & 10 & 20 & 16 & 8 & 21 \\
\hline 4 & 4 & 5 & 7 & 11 & 12 & 10 & 23 & 33 & 38 \\
\hline 5 & 2 & 5 & 0 & 2 & 3 & 2 & 18 & 57 & 40 \\
\hline Totals & 39 & 11 & 12 & 18 & 25 & 32 & 69 & 103 & 106 \\
\hline
\end{tabular}

Entries in the body of the table are pecks of three birds pooled.

The findings do raise the question of what conditions are necessary for obtaining Hess's results. One possibility is the mounting of the prisms. From Hess's photographs, it would appear that his prisms were not mounted in the frontal-parallel plane, but at an angle. The effect of this might have been to introduce a greater distortion of the visual field than should nominally have been present. We took great care with our hoods to mount them as near as possible in the frontalparallel plane.

Whether or not the mounting of the prisms is a factor, the question of whether chicks can or cannot compensate for distortion in their visual space remains open to further investigation.

\section{References}

HESS, E. H. Space perception in the chick. Scient. American, 1956 , $195,71-80$. 